\title{
Philosophiques
}

\section{Le rapport quantité-qualité chez Hegel et chez Bergson}

\section{Jean Theau}

Volume 2, numéro 1, avril 1975

URI : https://id.erudit.org/iderudit/203020ar

DOI : https://doi.org/10.7202/203020ar

Aller au sommaire du numéro

Éditeur(s)

Société de philosophie du Québec

ISSN

0316-2923 (imprimé)

1492-1391 (numérique)

Découvrir la revue

Citer cet article

Theau, J. (1975). Le rapport quantité-qualité chez Hegel et chez Bergson.

Philosophiques, 2(1), 3-21. https://doi.org/10.7202/203020ar d'utilisation que vous pouvez consulter en ligne.

https://apropos.erudit.org/fr/usagers/politique-dutilisation/ 


\title{
LE RAPPORT OUANTITÉ-QUALITÉ CHEZ HEGEL ET CHEZ BERGSON ${ }^{1}$
}

\author{
par Jean Theau
}

La philosophie contemporaine semble devenue, parfois, une affaire de scoliastes, dont les travaux de bibliothèque auraient usé les yeux et dont le regard, rendu myope, ne serait plus récompensé que rarement par une perception. A force de parler ou d'écrire sur le langage, de réfléchir sur la réflexion et d'argumenter sur des arguments, l'objet dont il était question à l'origine des débats, - car on veut croire qu'il y en avait un, - finit par se pulvériser, s'obscurcir et finalement disparaître à travers tant de microscopes interposés. Et avec l'objet, l'intention de l'analyse se dissipe aussi. Perdu dans l'immense océan des critiques et des analyses infinitésimales, on se prend à regretter l'abrupte simplicité du premier néo-positivisme, quand il s'agissait de soumettre la philosophie aux canons rudes mais salubres de la science expérimentale et de la logique, interprétées il est vrai en

1. Cet article a été donné comme conférence au Congrès annuel de l'A.C.P. à Toronto, mai-juin 1974. L'occasion en explique le genre. Nous voulions suggérer, développer et discuter une idée, celle des affinités et des contrastes entre deux grands philosophes sur un même problème, plutôt qu'ajouter un chapitre à l'histoire de la philosophie proprement dite. Ainsi avons-nous été conduit d'une part à réduire au minimum, dans notre texte écrit, les preuves référentielles, d'autre part à appuyer certains traits tandis que nous passions très vite sur quelques autres, qu'il eût fallu souligner davantage, si nous avions considéré isolément, ou plus analytiquement, chaque doctrine. Cependant, même après l'excellente discussion qui a suivi notre exposé, il ne nous semble pas qu'une seule de nos assertions se soit avérée peu fondée. C'est pourquoi, malgré leur caractère incomplet, nous osons les proposer ici au lecteur.

L'objection la plus forte nous a été adressée, nous semble-t-il, par Monsieur Yvon Lafrance. Comment comparer deux textes aussi différents que la Science de la Logique, où il est question de catégories et de dialectique logiques, et l'Essai sur les données immédiates de la conscience, où il est avant tout question de psychologie? Nous avons répondu que, de 
vue de très philosophiques négations. Cependant, pour essayer de recueillir quelque idée sur un problème qui ne devrait pas cesser d'être au centre de la philosophie contemporaine, puisqu'il commande, outre un bon nombre de questions métaphysiques, la théorie de la logique, des mathématiques et de la physique, ainsi que la méthodologie des sciences humaines, ce n'est pas vers le Wittgenstein du Tractatus ou le Cercle de Vienne que nous nous tournerons, mais vers deux géants de la pensée spéculative que, dans trop de quartiers intéressés par ces problèmes, on a sans doute tort de négliger. Car juchés sur leurs épaules, nous aurions quelque chance de mieux percevoir le paysage dans lequel nous vivons et où se développent nos sciences.

Apparemment, Hegel et Bergson n'ont guère en commun que le discrédit qu'ils partagent parmi les contempteurs de la métaphysique. Quoique Bergson lui-même, si ouvert à la pensée

l'aveu même de son auteur, la logique hégélienne est une logique très spéciale, qui nous plonge tout de suite et nous maintient en pleine métaphysique, et qui, précédée et éclairée comme elle l'est par la Phénoméno. logie de l'Esprit, fait à la réflexion épistémologique et même psychologique une large part. Réciproquement, la psychologie bergsonienne est une psychologie entendue à la manière de Maine de Biran et de Lachelier, c'est-à-dire ouvrant très vite sur des débats métaphysiques, et elle implique une critique des catégories majeures de l'entendement. C'est ainsi que l'Essai, comme nous l'avons montré dans notre Critique bergsonienne du Concept, associe toujours à l'étude des données immédiates une réflexion critique sur des notions, et en particulier sur les notions qui nous intéressent ici, celles de quantité et de qualité.

L'objection essentielle que nous nous adresserions à nous-même concernerait la théorie hégélienne de la qualité. Car s'il est vrai que pour Hegel comme pour Bergson la qualité se pose d'abord, elle est donnée puis posée, dans la Science de la Logique, comme le premier moment d'un concept et ce moment est quelque chose de plus abstrait que le second moment, représenté ici par la quantité. La primauté de la qualité ne revêt donc pas chez Hegel, du moins sans restriction, cette valeur de préséance ontologique qu'elle comporte incontestablement chez Betgson. Néanmoins si le premier moment d'un concept, en tant qu'il est pensé de façon catégorielle, est le plus abstrait, il est aussi d'une certaine manière, en tant qu'il se trouve à la source du mouvement conceptuel, plus tiche que le second moment, où se développe le travail du négatif. La qualité, - sous sa forme générale de Bestimmtheit sinon sous sa forme spécifique de Qualität, - constitue donc le fond de la logique du Sein et se retrouve ainsi partout dans less trois moments de cette sphère, alors que la quantité, d'origine négative, se greffe sur elle à partir du second moment seulement, pour s'y combiner étroitement au niveau du troisième. 
anglaise, semble avoir été plutôt imperméable à l'influence allemande, sa philosophie de l'intuition et de la vie, ajoutée à l'antirationalisme décidé qu'on lui prête, l'a fait rattacher parfois à Schelling, à Schopenhauer ou à Nietzsche, mais à Hegel jamais. Celui-ci, on le sait, prétend reconstruire la réalité avec des concepts, et il n'y a pas d'entreprise dont Bergson ait médit davantage. Selon le premier, le devenir est à la fois le moteur et le champ grâce auxquels se déploie la logique; liberté et nécessité se confondent; Dieu est atteint par l'achèvement du savoir rationnel. Pour le second au contraire, le devenir réel défie toute combinaison de catégories; il y a antithèse et conflit entre la liberté et la nécessité; Dieu se découvre à l'âme dans une intuition mystique qui transcende de beaucoup la raison. L'un affirme sans cesse que la philosophie doit viser au système et l'autre ne cesse de répéter qu'elle doit avant tout s'en garder. On multiplierait sans peine les oppositions. Cependant, lisant de près, il y a quelques années, la Science de la Logique après une étude approfondie du bergsonisme, nous étions frappé par d'importantes analogies et même par un grand nombre d'expressions similaires. En particulier, c'est en termes souvent très proches que les deux philosophes prennent leur distance vis-à-vis du kantisme, dont ils répudient ensemble la méthode ${ }^{2}$ et la doctrine pour restaurer la métaphysique. Et dans les deux phillosophies, cette dernière est présentée comme une appréhension de l'être à la lumière de la vie, ou comme une intelligence de leur identité profonde, alors que l'hostilité à la métaphysique fait figure de parti-pris pour les choses mortes. Dans les deux cas encore, une hostilité de ce genre, expression d'une impuissance, est rapportée aux limites de l'entendement, qui se prend orgueilleusement pour le tout de l'esprit, quand il n'en est qu'un moment ou une province. En sorte que, si on adoptait cette ligne de comparaison, c'est-à-dire si on suivait le progrès des deux pensées depuis leur réflexion sur la conscience jusqu'à leur méditation sur le christianisme, on verrait les affinités se multiplier elles aussi. Mais nulle part les analogies ne nous ont paru plus frappantes, ni les oppositions plus vives et plus directes, que sur le problème du rapport quan-

2. C'est ainsi que, sans citer Hegel, Bergson reprend à plusieurs reprises l'image de l'homme qui voudrait savoir nager avant de se mettre à l'eau, et conclurait de son incapacité à l'impossibilité de la natation. 
tité-qualité, rapport essentiel aux yeux des deux philosophes, qui lui ont consacré l'un et l'autre des développements très étendus. Comment semblable connexion s'est-elle produite et pourquoi l'a-t-on si peu remarquée ? Ce sont là deux questions qui pourraient tenter un historien, parce que leur étude jetterait peut-être quelque clarté sur le cheminement de la pensée au XIXe et au $\mathrm{XXe}$ siècle en Europe. Mais ici nous négligerons l'histoire pour ne nous attacher qu'à la doctrine. Sans trop nous appesantir sur les preuves textuelles de nos assertions, que nous nous bornerons à indiquer en note, nous essayerons de faire ressortir ce en quoi se rapprochent et ce en quoi s'opposent les deux façons dont Hegel et Bergson expliquent le rapport quantité-qualité. Enfin nous dirons quelques mots sur la perspective génétiste qui nous paraît commune aux deux penseurs et nous voudrions suggérer, d'un trait, la possibilité d'une autre approche.

\section{I - LES AFFINITÉS}

On notera tout d'abord des similitudes de vocabulaire qui sautent aux yeux, autant qu'elles surprennent, quand on lit l'un après l'autre deux textes aussi différents que le premier livre de la Science de la Logique et les deux premiers chapitres de l'Essai sur les données immédiates de la conscience. Les ressemblances apparaissent dès que les deux auteurs entreprennent de qualifier la qualité. Hegel la définit comme simple (einfach), immédiate (unmittelbar), comme un en-soi (an-sich) qui est cependant un être-pour-un-autre (das Sein-für-Anderes) et qui est essentiellement, quoique déterminée (bestimmt), variable (verànderlich) ${ }^{3}$. Or, bien que Bergson ne cherche jamais à parler allemand en français et, sans doute, n'ait nullement envie d'hégélianiser, il use, pour la même qualification de la qualité, de termes soit identiques soit très proches, puisque, pour lui aussi, la qualité est quelque chose de simple, d'immédiatement donné à la conscience, de réel autant que de perçu, donc en-soi autant que pour-soi, une détermination indivisible et absolue mais pourtant fondamentalement reliée à d'autres par un rapport d'hétérogénéité, enfin la variation même, car du fait qu'elle dure elle change. La similitude frappe encore davantage quand on passe au domaine de la quantité. Ici, ce ne sont plus seulement des mots, ce sont des expressions entières qu'il faudrait citer. Sans 
doute, Bergson ne dira guère que la quantité est «la qualité supprimée ou niée », car il n'a pas de goût pour la dialectique de la négation. Toutefois, avant même qu'il ne montre dans la quantité matérielle une «inversion» du devenir qualitatif (cf. Evolution créatrice chap. III), il affirme que l'unité qui est au principe du nombre a été obtenue par un «effacement» des différences qualitatives, lequel effacement a pour résultat une absolue identité ; on est vraiment très proche de «l'un » hégélien (das Eins), défini par l'exclusion de toute détermination (es ist unbestimmt) ${ }^{4}$, la reproduction indéfinie de soi (Das Eins ist somit Werden zu vielen Eins) ${ }^{5}$ et la parfaite identité à soi (die Eins dasselbe). Des deux côtés, la multiplicité qui constitue le nombre, et d'ailleurs la quantité en général, s'engendre par la répétition du même (ein Wiederholen von einem und eben demselben), se caractérise par la juxtaposition et l'extériorité de ses membres (die Äusserlichkeit des Quantums; die Quantität ist Âussereinandersein an sich) ${ }^{7}$. Des deux côtés également, les opérations arithmétiques sont tenues pour des opérations analytiques (ein Synthesieren, des aber gänzlich analytischer $\mathrm{Na}$ tur ist), l'augmentation et la diminution quantitatives sont opposées au devenir concret (ist dies aber nicht sowohl ein Werden) ${ }^{\text {s. }}$ La quantité n'a pas assez de consistance pour évoluer; composée d'éléments homogènes et stables (gleich, stetig, spröde) sinon immobiles et morts (bewegunglosen, toten) elle est certes indéfiniment modifiable mais demeure privée, en elle-même, de dynamisme ${ }^{9}$.

3. Toutes ces expressions sont empruntées à la Wissenschaft der Logik I Suhrkamp Verlag, Frankfurt am Main 1969. (W.d.L.I.) Cf. DAS DASEIN en particulier, 'p. 117-118-124-127-128-129-135. ('Toutes nos références renverront à cette édition). Voir aussi p. 209 Die Grösse (Quantität) : «Die Qualität ist die erste, unmittelbare Bestimmtheit, die Quantität die Bestimmtheit, die dem Sein gleichgültig geworden, eine Grenze, die ebensosehr keine ist. »

4. W.d.L.I. p. 183. cf. aussi « es ist unveränderlich 》

5. W.d.L.I. p. 187.

6. W.d.L.I. p. 191.

7. W.d.L.I. p. 260, p. 234 et 228 et d'ailleurs tout le texte p. $210-213$.

8. W.d.L.I. p. 238 et p. 187.

9. W.d.L.I. p. 246 : « Die weiteren, konkreten, wahren Gedanken, das Lebendigste, Beweglichste, nur im Bezichen Begriffene, in dieses Element des Aussersichseins selbst, werden zu toten, bewegungslosen Bestimmungen ». $\mathrm{L}_{\mathrm{a}}$ plupart des termes français dont nous nous sommes servi ici pour carac- 
Toutefois, pour apprécier comme il convient l'étendue des similitudes autant que la netteté des différences entre les deux doctrines, il faut dépasser le niveau du vocabulaire et pénétrer jusqu'à l'économie des pensées, c'est-à-dire jusqu'à leur ordre et leur contenu. Or un fait d'importance capitale en philosophie caractérise tout d'abord la position de nos deux auteurs. L'un et l'autre accordent à la question quantité-qualité une portée ontologique qu'elle n'avait plus conservée depuis Aristote et les médiévaux. Ce qui signifie qu'en réfléchissant sur ces rapports, non seulement on éclaire de façon décisive la structure de la perception sensible et du savoir, mais encore on avance profondément dans l'intelligence de l'être tel qu'il est en soi. Ainsi la théorie de la qualité et de la quantité nous introduit directement à la théorie de la substance ou de la réalité, elle décrit, en le divisant d'après une différence essentielle, tout l'aspect phénoménal de la substance, autant qu'elle analyse en ses catégories fondamentales l'ensemble du donné perçu par la conscience. Qu'on nous entende bien. Nous ne voulons pas insinuer que, de Descartes à Kant, le problème des rapports quantité-qualité ait été négligé. Il était au coeur de la physique, de l'épistémologie et de la métaphysique cartésiennes. Locke le reprenait, après Galilée, Hobbes et Boyle, par sa distinction fameuse entre qualités premières et qualités secondes. Toute la pensée de Leibniz, dans ses aspects multiformes, en était préoccupée; celle de Malebranche également. Et Kant, après avoir restitué à la quantité et à la qualité leur rôle de catégories logiques primitives, y rattachait, par «les axiomes de l'intuition» et «les anticipations de la perception », la connaissance a priori de la nature. Inversement il n'est pas douteux que Hegel et Bergson ont retenu l'un et l'autre l'approche phénoméniste qui, en ce domaine aussi, avait prévalu depuis Hume et Berkeley, sinon depuis Leibniz et Locke. Car pour la Science de la Logique et pour l'Essai, qualité et quantité sont des données de la conscience avant d'être des états ou des propriétés de la substance et les deux termes sont analysés, à l'encontre de ce qui se passe chez Aristote, sans référence à

tériser la quantité et la qualité sont employés par Bergson (cf. notamment le Ile chap. des Données Immédiates p. 51-66: La multiplicité numérique et l'espace - L'espace et l'homogène, et chap. I, p. 5-7: L'intensif et l'extensif : c'est pourquoi nous avons voulu éviter la répétition fastidieuse des guillemets et nous nous sommes borné à mettre à la suite l'équivalent hégélien. 
cette dernière, comme si leur consistance phénoménale suffisait à leur existence en soi. C'est que, chez les deux auteurs, la séparation entre le phénomène et l'être a cessé. L'être concret, parce qu'il est d'abord devenir et que le devenir implique un élément de conscience (concept chez Hegel, mémoire et tendance chez Bergson), s'est rapproché du phénomène. Mais le phénomène, parce qu'il est l'unité indivisible d'une forme et d'un contenu, manifeste à nouveau l'être même. Ainsi quantité et qualité ne sont plus l'une le mode précis de l'être matériel l'autre le mode confus de la subjectivité comme cela tend à se produire chez Descartes, ou deux formes que le sujet transcendantal impose à l'expérience comme chez Kant. Chacune d'elle représente une région de l'être, ou plus exactement une manière d'être, une spécification objective ${ }^{10}$.

Autre point commun d'importance: la qualité redevient ou devient première par rapport à la quantité. Aristote, on le sait, avait hésité sur la relation hiérarchique qu'il y aurait lieu d'établir entre les deux termes, ou plutôt il ne s'était pas prononcé nettement. D'une part, la qualité est pour lui une disposition qui affecte la forme des choses, ou du moins leur matière en tant qu'elle a déjà acquis quelque forme et ainsi se prête au changement. La quantité au contraire, du moins en tant que multiplicité ou homogénéité indéfinie (car il n'en est pas tout à fait de même en tant que grandeur) ${ }^{11}$, se tient plus près de la matière. Ainsi, dans la mesure où la forme est plus noble que la matière, la qualité est elle aussi quelque chose de plus noble - entendez également de plus déterminé et de plus intelligible - que la quantité. D'une manière très différente, mais tout aussi nette, Descartes et Kant ont suivi Aristote sur ce point: pour eux, la quantité prévaut sur la qualité ou la précède (mais cette fois la précède aussi en intelligibilité) dès qu'il s'agit de définir la structure de l'univers physique. Et il est remarquable qu'un Renouvier et un Hamelin, pour faire la théorie catégorielle d'un monde

10. Même si ce terme semble mieux convenir à la pensée de Hegel quà celle de Bergson, telle qu'elle s'exprime dans l'Essai, il reste que ce dernier a voulu montrer, au chap. IV de Matière et Mémoire, que la qualité n'est guère moins objective que la quantité.

11. Ce qui fait songer à la différence hégélienne entre Grösse et Quantität, ou plutôt à la différence entre reine Quantität et Quantum. 
qu'ils jugent fini, voudront rétablir cette préséance ontologique, corollaire au demeurant d'une infériorité axiologique. Ce dernier cas mériterait d'être étudié de près, car Renouvier et Hamelin adoptent précisément le point de départ qui a entraîné Hegel et Bergson au renversement du rapport, à savoir un point de départ phénoméniste. Mais aux yeux des premiers les catégories constituent essentiellement, en tout cas premièrement, des moments de la pensée, tandis que pour Hegel elles forment aussi simultanément et pour Bergson représentent fondamentalement soit des moments soit des aspects de la réalité. Or une réalité qui se réduirait à la quantité serait condamnée à se dissoudre et même, en vérité, ne saurait se constituer : elle tomberait en poussière évanescente avant que d'être née. Quoi qu'on fasse, comme Hegel l'observe à l'encontre de Kant, le composé, pour être, suppose le simple, le rapport d'extériorité quelque intériorité et l'homogène, pour se multiplier ou se diviser, quelque différenciation. Enfin le nombre même, pour ne rien dire de l'étendue, implique quelque ciment unificateur et la quantité, dont l'unité se prête à toutes les limites ${ }^{12}$, n'a pas de consistance en elle-même. C'est pourquoi dans l'ordre de l'être aussi bien que dans celui du connaître, dès qu'il ne s'agit plus de pures formes abstraitement considérées mais encore d'un contenu, la qualité se pose d'abord. Elle seule fournit un véritable immédiat, «le fond sur lequel viennent se greffer toutes les déterminations》 (Science de la Logique Trad. Jankélévitch p. 106). La quantité au contraire a pour condition et origine de la qualité transformée; par nature et position elle est quelque chose de médiat.

Il est une troisième similitude capitale, sur laquelle il vaudrait la peine de consacrer des développements considérables et d'ailleurs nuancés, à la mesure de ceux que les deux philosophes ont eux-mêmes consacrés à cette question: il s'agit de l'idée que l'on peut se faire des mathématiques et de la logique, de leurs rapports mutuels, de leur place dans la connaissance, de leur portée vis-à-vis du réel. Ici encore, nous serons très succinct, et par conséquent très sommaire. Mais une analyse très fine ellemême, donc plus sensible aux divergences, ne pourrait s'empê-

12. Cf. l'expression si fréquente dans la Science de la Logique, à propos du quantum : «gleichgültig gegen seine Grenze » et les expressions similaires. 
cher de constater qu'en dépit d'une théorie diamétralement opposée du concept, les deux philosophes se rejoignent pour affirmer ensemble, contre Kant et Descartes, qu'il n'y a pas d'opposition essentielle entre les mathématiques et la logique (entendons, pour Hegel, la logique de l'entendement) puisque dans l'une et l'autre discipline on demeure sous la domination du principe d'identité, on a affaire à des ensembles dont les membres restent extérieurs les uns aux autres sinon juxtaposés, par suite à des synthèses qui ne sont pas de vraies synthèses mais simplement des analyses remembrées, et parce que dans les deux cas l'usage de symboles représentatifs caractérise non seulement l'expression de la pensée mais encore sa véritable forme. Ainsi, bien loin qu'une mathématique universelle, c'est-à-dire une science unique dont la mathématique serait l'âme et le modèle, soit possible et souhaitable, l'intelligence de type mathématique ne représente qu'un moment ou une direction de la connaissance, moment et direction étroitement solidaires d'un objet que le réel déborde infiniment. Bref, la mathématique et la logique ne nous donneront jamais la clef de l'aspect dynamique des choses; dès qu'une réalité se manifeste, même sous la forme de minuscules quanta, sa consistance et son pouvoir leur demeurent cachés; encore moins peuvent-elles comprendre l'organisation, la vie, la conscience ${ }^{13}$, et ce serait une catastrophe si l'humanité, négligeant l'enseignement de la philosophie, venait à s'imaginer qu'elles épuisent l'esprit.

\section{II - LES CONTRASTES}

La différence essentielle des deux doctrines provient tout d'abord des méthodes qui leur sont liées et de ce que celles-ci présupposent. Hegel, on le sait, est intéressé par le concept et par le développement rationnel de celui-ci suivant une méthode dialectique, parce qu'il voit dans le concept ainsi développé le tout de l'être et de la vérité. N'oublions pas d'autre part - car cette position de principe s'en trouve accentuée - que Hegel traite du rapport quantité-qualité, comme d'ailleurs l'avait fait Aristote, au niveau de la logique, c'est-à-dire que, en dépit de

13. Lesquelles deviennent des choses «mortes et immobiles», disent ensemble Hegel et Bergson, lorsqu'on les plonge dans cet élément de l'extériorité (in dieses Element des Âussersichseins). 
ses thèses sur l'immanence de la forme et du contenu, il envisage le rapport des notions plutôt que celui des phénomènes concrets qui leur correspondent: ceux-ci sont évoqués certes, et parfois longuement, surtout dans le cas de la quantité (le concret de la qualité avait été étudié davantage dans la Phénoménologie, lorsqu'il était question de la certitude sensible, et le sera à nouveau dans La Pbilosopbie de la Nature), mais à titre d'illustration seulement. Le philosophe allemand sera donc préoccupé avant tout de situer les deux notions dans la hiérarchie des concepts, puis de les mettre en relation l'une avec l'autre, ainsi qu'avec les notions antécédentes et subséquentes, en les analysant selon le procédé dialectique. Il s'ensuit que qualité et quantité sont placées ensemble dans l'ordre de l'être, entendons de l'être qui se développe en être-là (Dasein) et en quelque chose de fini, qui n'est par conséquent que le premier moment de la réalité et de la vérité, qui enfin absorbe ou retient une conscience qui ne s'en est pas encore dégagée par le retour à soi de la réflexion et l'explication du concept. En d'autres termes, il s'agit de l'être tel que sans doute, dans des moments ultérieurs, l'entendement l'analyse et la raison le comprend, mais premièrement tel que la conscience sensible l'appréhende ${ }^{14}$. Le niveau de l'être constitue, dans le développement de ses déterminations, le premier résultat du devenir (Aus dem Werden das Dasein hervor), lui-même engendré par la médiation réciproque de l'être et du néant (Einheit des Seins und Nichts). Or si on comprend d'une part que chaque détermination de l'être porte en elle sa négation et que la contradiction qui en résulte doit être résolue par une négation de la négation laquelle pose une affirmation enveloppante (la célèbre Aufhebung), si on comprend d'autre part que l'en-soi, en passant par la scission de l'être-pour-un-autre, cherche à s'accomplir en pour-soi, si enfin on sait appliquer cette double dialectique à l'analyse de la qualité et de la quantité, on verra la seconde se préparer dans le développement de la première, puis retrouver à son tour la première, jusqu'à ce qu'elle la ressaisisse tout entière, au niveau supérieur de la mesure, par son propre développement. Ou plus précisément, le fini qualitatif

14. Tandis qu'elle n'accède pas encore au niveau de l'essence et encore moins au niveau du concept, et bien que le concept soit nécessaire pour faire la théorie de cette conscience. 
tend à passer dans son opposé, l'infini, mais, sur le plan de l'être immédiat où il se meut, il ne trouve cet infini que dans «l'un» abstrait, qui n'est la supression du rapport à l'autre que dans la forme de l'extériorité ${ }^{15}$, c'est-à-dire par l'exclusion de toute détermination. Et ainsi la qualité, en s'exacerbant, engendre la quantité, laquelle nous ramène tout d'abord à la finitude et à "la mauvaise infinité », pour ne rejoindre l'infini véritable qu'en devenant rapport défini, c'est-à-dire en produisant en son sein de la qualité.

Tout ce mouvernent conceptuel, si l'on excepte les brillantes analyses auxquelles il a donné lieu, devait être sans nul doute considéré par Bergson comme dialectique, mais au sens le plus péjoratif qu'un Aristote ou un Kant eussent pu attacher à ce terme. Car, pour l'auteur de l'Evolution créatrice, les concepts résultent de points de vue plus ou moins pragmatiques sur les choses, qui en tout cas sont données antérieurement et dont ils ne fournissent que des représentations partielles. Comme l'aspect représenté de la chose a un lien direct avec l'aspect qu'on néglige ou qu'on ignore, on pourra, en passant d'un concept à son opposé, ainsi de la droite à la gauche, de la surface au volume, du discontinu au continu, passer d'une partie de la réalité à une autre. On aura ainsi l'impression qu'on avance, dans la connaissance des choses, par un mouvement logique de la raison ${ }^{16}$. Et pour peu qu'on ait reçu le génie de la dialectique - or Hegel le possédait à un degté exceptionnel - on systématisera le procédé en montrant que tout concept tend à passer dans son opposé par suite de son insuffisance, c'est-à-dire au fond en raison de sa nature abstraite, et on verra dans la puissance du négatif le moteur commun des choses et de la pensée. Mais il s'agit là, pense Bergson, d'un raisonnement doublement illusoire. En fait, la force progressive n'appartient pas aux concepts mais à l'expérience, que les concepts essayent péniblement et maladroitement de rejoindre, en l'épelant morceau par morceau, parce que la

15. L'infini hégélien se définit en effet (et même sur le plan de la quantité cf. les notes de la Logique concernant le calcul infinitésimal), non par la grandeur, mais par la perfection de l'achèvement et de la complétude, c'està-dire par un rapport à l'autre devenu intégralement rapport à soi.

16. Tout ce processus se trouve décrit par Bergson, sans être spécialement appliqué à Hegel, dans l'Introduction à la Métaphysique. 
réalité s'y trouve donnée, de par sa présence et virtuellement, tout entière. En outre, la dialectique conceptuelle, toujours et partout uniforme autant que superficielle, nous cache et la nature profonde et la genèse spécifique des choses, sur lesquelles nous ne saurions jamais rien de vrai sans consulter encore, avec l'attention la plus pénétrante et la plus ouverte à la fois, l'expérience. Or l'expérience, dès qu'elle dépasse les cadres de l'habitude, produit de l'inattendu, car elle a sans cesse affaire à de la contingence et à des créations, et ne ramènera pas la raison, contrairement à ce que Hegel pensait, vers les formes a priori de la dialectique. Son contenu n'est pas si docile au concept, qui n'a guère plus le don de l'anticipation ${ }^{17}$ que le don prophétique, et si le concept pouvaî́ être figuré par l'oiseau de Minerve, ce serait bien le cas de dire que, pour annoncer la vérité concrète, «il se lève toujours trop tard ».

C'est pourquoi, quand il voudra préciser à son tour le rapport quantité-qualité, Bergson ne se fiera pas à la dialectique. En vérité, le philosophe est parti d'un état de fait assez sembilable à celui qui avait dû éveiller sur bien des points la réflexion critique de Hegel, qui en tout cas allait provoquer intensément la sienne. Car les mises en garde et les développements de la Science de la Logique semblent avoir eu peu d'effet sur le développement des sciences positives et, dans la deuxième moitié du XIXe siècle, elles avaient même perdu beaucoup de leur influence sur la philosophie. Le «retour à Kant» se conjuguait avec un positivisme beancoup plus sommaire que celui d'Auguste Comte et avec un évolutionnisme attiré par le matérialisme, pour remettre à l'ordre du jour, bien au-delà de ce que Kant et Auguste Comte eussent eux-mêmes admis, le rêve d'une mathématique universelle. Une fois de plus, la quantité bousculait la qualité, ou plutôt l'envahissait et prétendait la soumettre: Bergson luimême, en ses jeunes années, semble avoir partagé ce rêve. Mais voici qu'aux yeux d'une réflexion plus attentive, la durée vécue, qu'il fallait bien en définitive appeler la durée réelle, puisque le temps de la représentation mathématique n'est que de l'espace

17. Sauf peut-être dans le champ de l'espace et de la réalité matérielle, une fois que ceux-ci sont donnés, parce qu'ils sont le lieu de la similitude et de la répétition. 
déguisé, résistait à l'invasion et refusait l'intégration. Or avec la durée, c'était sa substance qualitative qui ne se laissait pas transformer ou même traduire en quantité. Que faire alors, devant ce fait indubitable, pour répondre à la fonction de vérité intégrale ou absolue que doit assumer la philosophie? Délimiter les concepts l'un par l'autre, expliquer leur passage l'un dans l'autre (avec les confusions qui en résultent) et cependant maintenir leur irréductible distinction? C'était là, on l'a vu, la méthode hégélienne. Mais Bergson ne songera pas un instant à l'employer. Une tentation aussi vive, aussi permanente au cours de l'histoire, que la traduction de la qualité en quantité devait avoir, à ses yeux, une origine autrement profonde qu'une simple confusion de concepts : il fallait la chercher dans les habitudes ancestrales de la vie et de la pensée. Et puisqu'il y avait lieu de redélimiter les concepts, il convenait de le faire en les renvoyant à leur source et à leur objet, c'est-à-dire au domaine empirique avec lequel, pour des raisons d'abord pratiques, ils sont censés s'accorder. En suivant cette voie, la réflexion philosophique devait s'apercevoir que les diverses notions comprises dans l'idée de quantité ont pour objet réel l'espace, ou tout ce qui relève essentiellement de l'espace, et pour finalité première notre action sur la matière. Sans doute l'espace géométrique est quelque chose de plus que la quantité pure et simple, telle que l'envisage par exemple l'algèbre, et l'étendue matérielle a un contenu qualitatif irréductible à l'espace géométrique. Il faudra donc se demander quelque jour ${ }^{18}$ en quoi consiste ce plus que contient l'étendue matérielle et d'où il provient, de même qu'on devra préciser le mécanisme et le sens du processus grâce auquel l'intelligence humaine progresse dans l'abstraction logico-mathématique. Mais le concept de la quantité — ni d'ailleurs aucun autre - ne nous instruira de lui-même, pour autant qu'on l'analyse ou qu'on le dialectise, sur le progrès de la pensée logico-mathématique, encore moins sur la genèse de la matière. Pour résoudre conjointement l'une et l'autre question, il sera nécessaire d'avoir éclairé, dans la mesure où on le peut, l'évolution de l'homme d'une part, l'évolution de la nature d'autre part. De même, pour penser

18. Ce que Bergson a fait dans Matière et Mémoire (chap. I et IV) et dans l'Évolution créatrice (chap. III). 
la qualité, il faudra la saisir et l'approfondir dans la véritable source qui nous la donne, à savoir la conscience, et, au lieu d'essayer d'en exprimer par un concept les traits généraux, la tâche qui s'impose sera d'en bien discerner les différents types ${ }^{\mathbf{1 9}}$. Alors il sera temps de chercher comment, non pas dans l'ordre des concepts, mais dans l'ordre de la réalité psychologique, physique et morale, la qualité en se renversant, c'est-à-dire en se durcissant, en se répétant et en s'éparpillant, engendre soit la quantité elle-même, soit ce qui en suggère l'idée.

Sans nier pour autant qu'il y ait toujours de la qualité au coeur de la quantité et de la quantité au sein de la qualité, sans nier non plus qu'il y ait passage d'un terme à un autre, puisque par deux fois, au chapitre IV de Matière et Mémoire et au chapitre III de l'Evolution créatrice, il consacre de longues considérations à ce passage (toute qualité s'accompagnant d'une tension susceptible de se diffuser en extension quantitative, et toute quantité pouvant être convertie en qualité quand elle rencontre l'activité synthétique de la vie et surtout la capacité synoptique d'une perception), Bergson pense qu'en ce domaine comme dans les autres le rôle des concepts est essentiellement de retenir et de fixer les distinctions obtenues par l'analyse. Il y a sans doute passage naturel de la quantité à la qualité, puisqu'on voit par exemple des trillions de vibrations lumineuses se condenser, dans la perception oculaire, selon leur fréquence propre, en couleurs déterminées et un phénomène analogue se produit pour le son. Mais la médiation s'accomplit par l'intermédiaire du mouvement, d'un organe et de l'âme. Elle ne réside pas dans une contradiction intestine que les concepts pourraient révéler et développer asymptotiquement à l'infini, jusqu'au point où le passage brusque à un autre concept, hiérarchiquement supérieur, semble s'opérer dialectiquement de soi-même. Comme cette hantise de l'infini, même si elle est vraie, n'est pas observable, on la laissera de côté, et on cherchera d'abord à surprendre dans un phénomène réel de liaison, comme l'est la perception sensible par exemple, le passage d'un monde où la quantité domine, l'univers matérieil, à

19. Ce que Bergson entreprend de faire par exemple au chapitre I et II de l'Essai, au chap. I de Matière et Mémoire, et même si on étend assez le sens de ce terme, dans l'Évolution créatrice et les Deux Sources. 
celui que la qualité dirige, le monde des vivants. De même, c'est en réfléchissant sur la conscience et l'action des vivants que l'on parviendra à présumer, du moins par quelque lointaine analogie, comment l'intensité ou la tension qualitatives peuvent s'épancher ou s'affaisser en quantité. Mais la quantité, de par elle-même, n'engendrera jamais une qualité d'ordre supérieur. Pour cela il faudra, non pas «la patience du concept» - laquelle, pas plus que «la longueur de temps, ne fait rien à l'affaire»-, mais, et aux yeux de Bergson ce n'est pas la même chose, la patience inventive de la vie et le génie créateur de l'esprit. L'accumulation quantitative rassemble certes une puissance; mais cette puissance ne passe d'elle-mêrne à l'acte, ou par l'accident d'une étincelle, qu'à son niveau; pour l'élever, l'impulsion de quelque élan d'ordre vital est indispensable. L'énorme énergie solaire ne fait rien de vivant sur la terre sans la feuille verte.

\section{III - CONCLUSION}

Il ne saurait être question, en terminant une analyse déjà par trop sommaire, d'évaluer, avec une ambition de critique rigoureuse, les mérites et les démérites respectifs des deux théories que nous venons de confronter. Nous indiquerons seulement le sentiment d'ensemble que l'une et l'autre nous laissent, avant de dire un mot de l'hypothèse métaphysique que toutes deux, sur le problème en question se sont accordées à négliger et qui, cependant, apporterait peut-être quelque utile lumière à la raison.

Bergson, nous semble-t-il, n'a pas eu tort d'écarter la tentative dialectique de Hegel sur le plan où celui-ci, dans la Science de la Logique, a voulu l'opérer. Car nous ne pensons pas que Hegel ait réussi à engendrer dialectiquement la notion de la quantité à partir de la notion de la qualité et, à nos yeux, un abîme demeure entre la qualité que la quantité finit par retrouver en son sein et celle avec laquelle elle s'unit pour constituer la mesure (das Mass), la première étant d'ordre mathématique et la seconde, en définitive, d'ordre physique. L'un qui veut être absolument pour-soi (für-sich-sein) et exclure tout rapport à l'autre, diffère déjà énormément de toute notion que l'on peut se faire de la qualité ; et si l'on dit qu'il a été obtenu par l'action du négatif, on ne voit guère pourquoi la négation, qui a supprimé tout le reste, l'a épargné. De même l'un qui se repousse hors de 
lui-même ${ }^{20}$ pour former la multiplicité, puis qui s'attire ${ }^{21}$ pour la rassembler en nombre, ne ressemble guère davantage à l'unité vraiment calme et indifférente dont l'addition donne la série des nombres. Sans doute Hegel pourrait répondre que nous avons mal entendu sa dialectique, parce qu'elle n'est nullement pure synthèse, comme nous avons feint de le croire. Elle est tout aussi bien analyse en effet. La qualité va de la détermination à la diversité $^{22}$, et de la diversité à l'un, parce que l'en-soi n'est pas séparable de l'être-pour-un-autre, et celui-ci à son tour du poursoi ; car l'en-soi n'est que le moment d'un concept et tout concept comprend indivisiblement, dans son unité, avec l'en-soi l'êtrepour-un-autre et le pour-soi. Donc, quand par abstraction on pose l'en-soi isolément, on pose aussi virtuellement mais nécessairement la démarche vers le pour-soi, de même qu'en posant l'être on pose le néant puis leur réunion dans le devenir, et dans le devenir la nécessité d'un résultat. Admettons. Mais comment ne pas voir du même coup que toute cette démarche ne signifie rien, ne comporte pas la moindre possibilité, si on ne fait jouer au sein du concept une conscience intelligente qui divise les moments de l'abstraction, constate leur insuffisance respective et les réunit en un tout plus complet? Or cette conscience qui joue à d'intérieur du concept (et en vérité, en parlant de concept, Hegel se donne le droit de la faire jouer), on ne peut empêcher qu'elle ne déborde de beaucoup tout concept déterminé. Elle se souvient au moins confusément de son expérience entière et d'emblée elle a au moins une confuse idée de la totalité de l'être (l'überhaupt hégélien !). Dès lors, on ne peut guère s'empêcher de penser que c'est elle qui tire en avant la dialectique des concepts et lui fait accomplir les bonds de marche en marche et d'étage en étage, car elle a par avance aperçu tout l'escalier. Mais s'il en est ainsi pourquoi ne pas la faire entrer tout de suite en ligne de compte et ne pas chercher à surprendre en elle, dans son dynamisme effectif, la vraie genèse des concepts ? ${ }^{23}$ De la sorte, on se serait

20. Die Repulsion des Eins von sich selbst. W.d.L.I. p. 188.

21. Die Sich-in-ein-Eines-Setzen der vielen Eins ist die Attraktion. Ibid., p. 192.

22. Verschiedenheit.

23. Nous n'ignorons pas que Hegel avait adopté un point de vue similaire dans la Phénoménologie; mais il a été conduit à l'abandonner trop tôt, parce que déjà, dans cet ouvrage, le devenir de la conscience était dominé par une architecture logique. 
épargné un jeu futile, dont le déroulement artificiel risque fort de nous masquer les véritables rapports des choses et des pensées.

La méthode bergsonienne, qui d'emblée consent à l'expérience et fait à la conscience la part qui leur revient, c'est-à-dire la première, et qui ne sépare jamais les concepts ni de leur objet ni de leur source, nous parait donc sur ce point de beaucoup préférable. Par suite de quoi, la pensée bergsonienne nous instruit beaucoup plus, à nos yeux, que l'hégélienne, tant sur la distinction réelle de la quantité et de la qualité que sur le passage effectif de l'une à l'autre. Cependant, parce que Hegel fait plus confiance aux capacités analytiques et synthétiques du concept, il obtient assez souvent dans sa réflexion des clartés et des profondeurs que Bergson n'atteint pas. Ainsi, après que celui-ci eut séparé, dans l'Essai, comme l'avait fait d'ailleurs la Science de la Logique, la qualité de l'infini quantitatif ${ }^{24}$, il se préoccupe beaucoup d'en appréhender et d'en décrire finement l'expérience, mais on dirait qu'il hésite, presque qu'il s'interdit de la penser. En conséquence il n'y aura rien dans l'Essai qui approche des considérations hégéliennes sur la finitude de la qualité et son rapport à un infini qualitatif dans le devoir-être ${ }^{25}$. Or une telle limite et un tel rapport importent sans doute autant à la conscience que la coalescence des qualités au sein de la durée concrète! Comme des rapports analogues se retrouvent dans le domaine de la quantité et que les deux catégories enveloppent en quelque sorte toute la matière de l'expérience, il est à présumer que cette analogie de rapport, en dépit d'une différence radicale de contenu et de structure, doit présenter quelque importante signification soit dans l'ordre du logos, soit dans celui de l'être, soit dans les deux. Et si Hegel, à notre avis, n'a pas réussi à engendrer l'idée de la mesure à partir des simples notions de la quantité et de la qualité, l'intelligence qu'il avait acquise du réel, en méditant sur ces notions, l'avait admirablement préparé à saisir l'importance de la mesure dans le monde physique et, sous d'autres traits, dans le monde moral. Or Bergson est faible sur ce point : il considère longuement, et fréquemment, la mesure comme technique opé-

24. Nous faisons allusion, entre autres, à la célèbre critique bergsonienne des arguments de Zénon, que Hegel ne manque pas lui non plus d'évoquer.

25. Cf. les développements intitulés «Die Schranke und das Sollen 》 dans Wissenschaft der Logik - Suhrkamp p. 142-148. 
ratoire. Mais, parce qu'ici encore il faudrait dépasser l'expérience par le concept, on dirait qu'il craint de parler de la mesure qui informe la nature et règle la vie.

Un mot pour finir de l'hypothèse que nous aimerions envisager. En effet, malgré la gravité des enjeux et le caractère absolu des objets visés, il nous semble que la métaphysique ne se dégrade pas en passant, au moins pour commencer, par le mode hypothétique. Après tout, elle aussi vit d'idées et l'idée se présente toujours, au moins dans l'esprit humain, sous la forme d'un possible. Sans peut-être s'en apercevoir clairement, Hegel et Bergson ont accepté, au point de départ de leur réflexion, un certain nombre de présupposés que la philosophie du XIXe siècle avaient rendus en quelque sorte apodictiques. Et d'une certaine façon, ces présupposés ont commandé la solution que chacun d'eux propose pour le problème du rapport quantité-qualité. Dans l'ensemble, la philosophie du XIXe siècle, qui se voulait post-critique, s'était accordée pour écarter la notion de substance comme obscure et trop problématique. Par suite on pensait que les notions de quantité et de qualité, jointes à la notion de devenir et à celle d'ordre, devaient suffire à représenter sinon la forme, du moins toute la matière de la connaissance. Dès lors, en quoi pouvait bien consister la métaphysique de la quantité et de la qualité, sinon en un effort pour établir directement leurs relations réciproques ou pour engendrer l'un des deux termes à partir de l'autre, comme l'a manifesté la querelile un moment à la mode entre génétistes et nativistes? En vérité, c'est bien à une tentative de ce genre que Hegel et Bergson, en dépit de l'opposition radicale de leurs méthodes et parfois de leurs conclusions, se sont essayés tous les deux. Mais supposons, comme une longue tradition judéo-chrétienne et classique nous invite à le croire, que le monde auquel nous appartenons, quelle que soit son accablante immensité et son insondable ancienneté, soit un monde créé. Alors, ni la quantité ni la qualité ne constitueraient les premiers termes dont une théorie métaphysique des objets de l'expérience devrait partir. Tout essai de genèse qui voudrait dériver l'un de ces termes de l'autre serait vain et même faux dès le principe, autant qu'il se révèle fragile, en vérité, devant la critique. Car le concept dont la métaphysique d'un monde créé devrait s'inspirer 
serait le concept de l'être fini comme existant possible, puis comme existant réel. C'est à ce concept, envisagé sous ce double rapport, qu'il faudrait rattacher les notions de qualité et de quantité, ainsi que la vaste expérience et la pensée qui y correspondent. Quantité et qualité apparaîtraient de la sorte non comme les étapes ou les moments successifs d'une genèse, mais fondamentalement comme les résultantes simultanées d'une création. Et c'est dans leur relation à l'Esprit créateur tout d'abord, aux esprits créés ensuite qu'il conviendrait de chercher directement, pour autant que l'oeil de l'homme ne se perd pas dans ces profondeurs, leur raison d'être et l'explication de leur essence.

Université d'Ottawa 